\title{
The antioxidant activity and hypolipidemic activity of the total flavonoids from the fruit of Rosa laevigata Michx
}

\author{
Yue-Tao Liu, Bi-Nan Lu, Li-Na Xu, Lian-Hong Yin, Xiao-Na Wang, Jin-Yong Peng*, Ke-Xin Liu
}

College of Pharmacy, Dalian Medical University, Dalian, China; jinyongpeng2005@163.com

Received 21 December 2009; revised 8 January 2010; accepted 30 January 2010.

\begin{abstract}
In the present work, the antioxidant activity in vitro and hypolipidemic activity of the total flavonoids (TFs) from the Rosa laevigata Michx fruit was evaluated, and the antioxidant effect in vivo was also discussed. The TFs exhibited a high scavenging effect on 2, 2-diphenyl-1picrylhydrazyl (-DPPH) with $\mathrm{IC}_{50}$ values $0.01 \mathrm{mg} / \mathrm{mL}$, and a stong reduce power in the test. Hyperlipemic mice were intragastric administrated with TFs (25, $50 \mathrm{mg} / \mathrm{kg} /$ day) for 4 weeks, and fenofibrate was used as the positive reference substance. After the experiment, the levels of TC (total cholesterol), TG (triglyceride), LDL- C (low density lipoprotein-cholesterol) of the mice administrated with high-dose of TFs were markedly declined by $45.02 \%, 33.86 \%$ and $73.68 \%$, respectively, while HDL-C (high density lipoprotein-cholesterol) was significantly increased compared with model group. To investigate the hepatoprotective effect, histopathological assay, ALT (alanine aminotransferase), AST (aspartate aminotransefrase) and ALP (alkaline phosphatase) were also studied, and the results showed that TFs exhibited a favorable effect on liver protection, of which the levels of ALT, AST and ALP were elevated by $55.85 \%, 29.15 \%$ and $25.68 \%$, respectively. Furthermore, the TFs could significantly decrease the MDA (malondialdehyde) level and improve the levels of CAT (Catalas), SOD (superoxide dismutase), GSH (reduced glutathione), and GPX (glutathione peroxidase) compared with hyperlipemia mice. Our results suggested that TFs has a high antioxidant activity and hypolipidemic activity, which can be used as a potential medicine for cardiovascular diseases.
\end{abstract}

Keywords: Rosa laevigata Michx; Total Flavonoids; Antioxidant Activity; Hypolipidemic Activity

\section{INTRODUCTION}

Many studies have proved that reactive oxygen species (ROS) and free radicals play a vital role in maintaining human health. When the balance between the generating and scavenging of ROS and free radicals in vivo is destroyed, an oxidative stress would happen, which might lead to extensive oxidative damage to cellular biomolecules, such as DNA, proteins and lipids. Many chronic-diseases, such as hyperlipemia, hyperpiesia and cancer, have proved to be associated with the existence of oxidative stress.

Hyperlipemia is considered as a risk factor involved in the development of cardiovascular disease [1]. High lipid levels can harden the arteries or speed up the process of atherosclerosis. Nowadays, there are numerous hypolipidemic drugs for clinical use, however, the pharmacologists and chemists have been perplexing by the characteristic profiles of toxic side effects including numerous harmful syndromes [2,3], which can increase the risk of heart disease, stroke, and other vascular diseases. Thus, an investigation of hypolipidemic agents with negligible side effect seems important. In recent researches, there has been a growing interest in the ingredients of natural plants, vegetables and cereals, not only for their radical-scavenging activities, but also due to their neglectable physical side effects. There are numerous herbal medicines exerting good hypolipidemic actions with few side effects in Asian countries. Plant materials have long been used as traditional medicines for the treatment of a wide variety of ailments and diseases, such as the polysaccharides from Lycium barbarum, Saponins in Ipomoea batatas tubers, total flavonoids of Litsea coreana leaf, the flavonoid-riched extract from Eugenia jambolana seeds. Vast numbers of plants have been shown to lower plasma lipid levels [4-7].

$R$. laevigata Michx is a famous medicinal plant in China, and its fruit is widely used as the invigorator, paregoric and astringent. Now chemical and pharmacological researches have demonstrated that this medicinal plant can cure hyperpiesia, chronic cough, and dermatogic disease, as well as inhibit experimental arterial sclerosis [8]. Flavonoids are primarily considered as the pig- 
ments responsible for the autumnal burst of hues and many shades of yellow, orange and red in flowers and food. The flavonoids have long been applied to possess anti-inflammatory, antioxidant, antiallergic, hepatoprotective, antithrombotic, antiviral and anticarcinogenic activities [9-11]. Up to now, the report about the antioxidant activity and hypolipidemic activity of the flavonids from this medicinal plant fruit was not discovered.

The major of the present work is to study the antioxdant activity and hypolipidemic activity of the total flavonoids (TFs) from the fruis of Rosa laevigata Michx on hyperlipemic mouse. In the test, we measured the diversity on biotical parameters on hyperlipidemic mice and the antioxidant effect in vivo. This is the first cover about it as far as we known.

\section{MATERIALS AND METHODS}

\subsection{Plant Material}

The dried fruit of $R$. laevigata Michx was purchased from Yunnan Qiancaoyuan Pharmaceutical Company Co. LTD (Yunnan, China), and authenticated by Dr. Yunpeng Diao (School of Pharmacy, Dalian Medical University, Dalian, China). Voucher specimen was deposited in College of Pharmacy, Dalian Medical University (Dalian, China).

\subsection{Chemicals}

$\mathrm{NaNO}_{2}, \mathrm{Al}\left(\mathrm{NO}_{3}\right)_{3}, \mathrm{NaOH}, \mathrm{FeCl}_{3}$, potassium ferricyanide, cholesterol, sodium cholate (biochemical reagent), trichloroacetic acid (TCA) and vitamin C (VitC) were purchased from Shenlian Chemical Company (Shenyang, China). Rutin was purchased from TCM institute of Chinese Materia Medica (Nanjing, China). 2, 2-diphenyl-1picrylhydrazyl ( DPPH) was purchased from SigmaAldrich (St. Louis, USA). Commercial kits used for determination of TC (total cholesterol), TG (triglyceride), HDL-C (high density lipoprotein-cholesterol), ALT (alanine aminotransferase), ALP (alkaline phosphatase), AST (aspartate aminotranse- frase), MDA (malondialdehyde), CAT (Catalase), SOD (superoxide dismutase), GSH (reduced glutathione), and GPX (glutathione peroxidase) were all purchased from Jiancheng Institute of Biotechnology (Nanjing, China). Fenofibrate was purchased from Laboratoires Fournier S.A. (France). All the chemicals were of analytical grade.

\subsection{High-Fat Diet and Animals}

High-fat diet was made according to the method depicted by Experimental methodology of pharmacology [12], containing normal pulverized food (97.5\%), cholesterol $(2 \%)$ and sodium cholate $(0.2 \%)$. The cake was cut into pieces and dried at room temperature for 3 days before feeding to mouse.
Male Kunming mice were obtained from the Experimental Animal Center of Dalian Medical University (Dalian, China). The animals, weighted $20 \pm 2 \mathrm{~g}$, were group-housed and kept in a regulated environment at 25 $\pm 1{ }^{\circ} \mathrm{C}$ and $60 \pm 5 \%$ relatively humidity under $12 \mathrm{~h}$ light/12 h dark conditions. The animals had free access to water and normal or high-fat diet.

\subsection{Preparation of TFs from the Medicinal Plant}

The fruit was ground into powder. Samples of $1.0 \mathrm{~kg}$ were weighted and mixed with $60 \%$ aqueous ethanol (solvent: sample $=8: 1, \mathrm{v} / \mathrm{w}$ ) for extraction. The process was refluxed in haven for two times and $2 \mathrm{~h}$ for each. The extracted solutions were filtered and evaporated to $1000 \mathrm{~mL}$ under reduced pressure at $60^{\circ} \mathrm{C}$. Then, $100 \mathrm{~mL}$ of the residue was added into a glass column $(4.0 \mathrm{~cm} \times 60$ $\mathrm{cm}$, contained $200.0 \mathrm{~g}$ D101 macroporous resin purchased from the Chemical Plant of Nankai University, Tianjin, China). The column was first washed by water $(600 \mathrm{~mL})$ to remove the un-desired compounds, and then the resin was eluted by $40 \%$ aqueous ethanol $(800 \mathrm{~mL})$ to elute the targets. The $40 \%$ aqueous ethanol elution was collected and evaporated under reduced pressure at $60^{\circ} \mathrm{C}$ to dryness, and $5.44 \mathrm{~g}$ powder was produced, which was stored in a refrigerator for subsequent experiments.

\subsection{Determination the Content of Tfs the Medicinal Plant}

The content of TFs was determined with a colorimetric method described by China Pharmacopeia [13].

\section{6. -DPPH Radical Scavenging Activity Assay}

Different concentrations $(0-1 \mathrm{mg} / \mathrm{mL})$ of the TFs solutions were produced by dissolving the samples in deionised water. Each sample solution $(2 \mathrm{~mL})$ was mixed with $2 \mathrm{~mL}$ of ethanolic solution containing $2 \times 10^{-4} \mathrm{mM} \cdot \mathrm{DPPH}$. The mixture was shaken vigorously and left to stand for $30 \mathrm{~min}$ in dark place, and then the absorbance was measured at $517 \mathrm{~nm}$ against a blank [14]. VitC was used as the positive control.

\subsection{Reducing Power Assay}

The reducing power was determined according to the literature with some modifications [15]. VitC was used as the reference compound.

\subsection{Experimental Procedure}

After 1 week of acclimatization to the home cage, the mice were randomly divided into five groups with each group containing 10 mice. Group I (controlled group): animals were fed the normal laboratory diet daily for 4 weeks; Group II (model group): mice were fed the 
high-fat diet daily for 4 weeks; Group III (positive group): mice were fed the high-fat diet plus fenofibrate (50 mg/kg/day, i.g.) daily for 4 weeks; Group IV: mice were fed the high-fat diet plus TFs $(25 \mathrm{mg} / \mathrm{kg} /$ day, i.g. $)$ daily for 4 weeks; Group V: mice were fed the high-fat diet plus TFs $(50 \mathrm{mg} / \mathrm{kg} /$ day, i.g.) daily for 4 weeks. During the experimental procedure, the treated animals were given sufficient normal or high-fat diet.

\subsection{Preparation of Biotical Samples and Protein Assay}

After the experiments, the mice were killed and then the blood was collected, and the liver was quickly removed. The collected blood was placed 30-40 min for clot formation, and then the serum was separated by centrifugation at $3000 \mathrm{rpm}$ for $15 \mathrm{~min}$. Each liver tissue was immediately rinsed with saline, blotted on filter paper, weighed and finally stored at $-70^{\circ} \mathrm{C}$ pending biochemical analyses. One part of liver was cut and put into a flasket containing $10 \%$ buffered formalin solution for the following histopathology analysis. The other part of the liver tissues was sampled quickly and washed with chilled normal saline water. $10 \%(\mathrm{w} / \mathrm{v})$ of tissue homogenate was prepared in cold normal saline, and centrifuged at $3000 \mathrm{rpm}$ at $4^{\circ} \mathrm{C}$ for $15 \mathrm{~min}$, and the supernatants were preserved for the next step assay. Lowry method [16] was employed to measure the protein concentration in the homogenate with bovine serum albumin as the standard.

\subsection{Histopathological Assay}

Liver tissues were fixed with $10 \%$ neutral formalin and embedded in paraplast. Tissue sections $(5 \mu \mathrm{m})$ were cut and stained by hematoxylin and eosin.

\subsection{Measurement of Biochemical Parameters and Hepatic Enzymes in Serum}

The levels of TC, TG and HDL-C in serum were determined using enzymatic kits according to the manufacture's instructions. The LDL-C was estimated by the

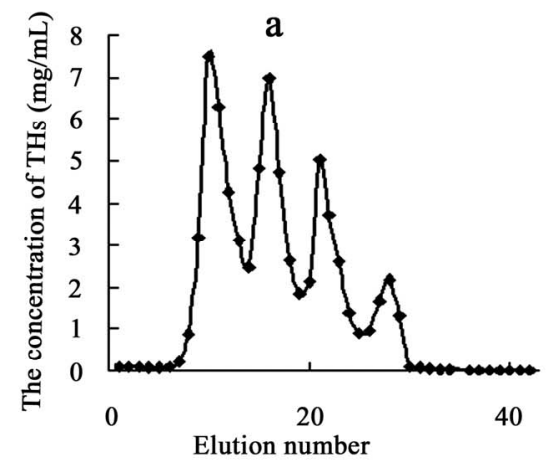

method of Friedwald et al. [17]. ALT, AST and ALP were all assayed using the corresponding commercial kits.

\subsection{Measurement of Hepatic Lipid Peroxidation and Antioxidant Enzymes}

The assays of the levels of MDA, CAT, SOD, GSH and GPX were measured following the kits' instruction.

\subsection{Statistical Analysis}

All values were expressed as mean \pm S.D. The significance of differences between the means of the treated and un-treated groups have been compared by one-way analysis of variance (ANOVA), followed by Student's $t$-test and $p$-values less than 0.05 were considered significant.

\section{RESULTS}

\subsection{Tfs Purification Protocol}

Before pharmacological investigation, the TFs from the fruit of R. laevigata Michx were required to prepare. In the present study, a kind of macroporous resin (MR) named D101, which has been widely used to purify flavonoids from medicinal plants as previously reported $[18,19]$, was selected to accomplish the work in this study.

In MR column chromatography, $100 \mathrm{~mL}$ residue of the extraction ( $1 \mathrm{~g}$ plant material $/ \mathrm{mL}$ ) was added into a glass column $(4.0 \times 60 \mathrm{~cm}$, containing $200 \mathrm{~g}$ D101 MR). Water $(600 \mathrm{~mL})$ and different concentrations of aqueous ethanol $(10 \%, 20 \%, 30 \%, 40 \%, 50 \%$, and $70 \%$, each 600 $\mathrm{mL}$ ) were used to elute the column in serials, and the elution solutions were collected individually to produce 42 different fractions (100 $\mathrm{mL}$ for each fraction) (Figure 1(a)). All the fractions were evaporated to dryness under reduced pressure at $50^{\circ} \mathrm{C}$, and then the contents of the

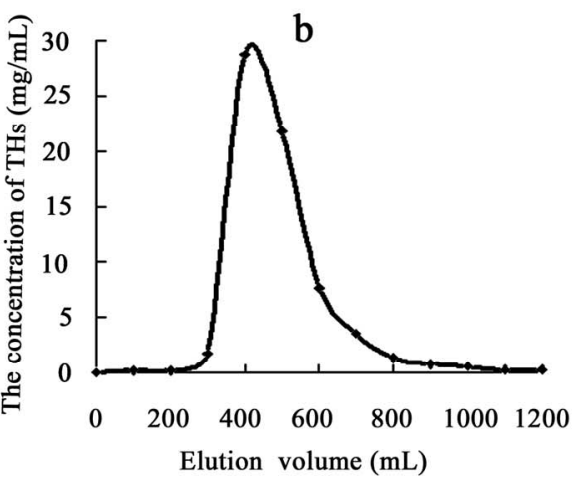

Figure 1. (a) The elution curve of the TFs on D101 MR column using water and different concentrations of ethanol as the elution solvents; (b) The elution curve of the TFs on D101 MR column using $40 \%$ aqueous ethanol as the elution solvent. 
TFs were determined. And then the recovery of every concentrations of alcohol was calculated through summing every fraction in one concentration. It was obvious that the flavonoids mainly existed in $10 \%, 20 \%, 30 \%$ and $40 \%$ aqueous ethanol elution solutions. There was nearly nothing in water, $50 \%$, and $75 \%$ aqueous ethanol solutions. Thus, water was first used to elute the water soluble chemicals, which were the undesired components and not supposed to be retained on D101 MR. Then, 40\% aqueous ethanol was selected to elute the targets.

Secondly, the required amount of water to remove undesired constituents and $40 \%$ aqueous ethanol to collect the TFs were detrmined. When water was applied to the column, each $100 \mathrm{~mL}$ was collected individually, and then the contents of the TFs in each fraction were detected. The results showed no flavonoid was eluted out untill water volume increasing to $600 \mathrm{~mL}$. However, with more water was used, part of flavonoids appeared in water. Therefore, $600 \mathrm{~mL}$ was set as the water quantity to clean the column in our research. Then, $40 \%$ aqueous ethanol was used to elute the flavonoids, and each $100 \mathrm{~mL}$ of the ethanol solution was collected individually and evaporated to dryness. The contents of the TFs were determined. As shown in Figure 1(b), it was apparently us that when $800 \mathrm{~mL}$ of $40 \%$ aqueous ethanol was used to elute the column, almost all the TFs was eluted out. Thus, it is reasonable to choose $800 \mathrm{~mL}$ as the elution volume.

After optimization, the TFs purification was carried out in triplicate as the mentioned protocol. The production rate, purity and recovery of the TFs were obtained, of which the production rate and the recovery were calculated as the following formula.

Production rate $(\%)=\frac{W_{1}}{W_{2}} \times 100 \%$, where $W_{1}$ represents the amount of the crude extract; $W_{2}$ represents the amount of plant material used for MR column chromatography.
Recovery $(\%)=\left(\frac{P \times W}{C \times V}\right) \times 100 \%$, where $P$ is the purity of the TFs in crude extract; $W$ represents the amount of the crude extract; $C$ is the TFs concentration in residue before MR column chromatography, and $V$ is the volume of the residue added into the column for purification. In the study, the production rate of the crude extract was $5.44 \pm 0.07 \%$, of which the content of the TFs reach to $78.45 \pm 2.41 \%$.

\section{2. -DPPH Radical Scavenging Activity}

DPPH, a stable free radical, has widely been used as a substance to evaluate the antioxidant activity of various samples. The method is based on the reduction of the absorbance of $\cdot$ DPPH solution at $517 \mathrm{~nm}$ in the presence of proton-donating substance, due to the formation of the diamagnetic molecule by accepting an en electron or hydrogen radical [20]. The TFs exhibted good scavenging activities to scavenge the stable radical DPPH to yellow-colored diphenyl picrylhydrazine in a dose dependent. As shown in Figure 2(a), the TFs showed strong scavenging activity on -DPPH with no difference compared with $\mathrm{VitC}$, and the estimated $\mathrm{IC}_{50}$ value was $0.01 \mathrm{mg} / \mathrm{mL}$. TFs can clear almost $90 \%$ of the radical at $0.15 \mathrm{mg} / \mathrm{mL}$. From our test, the TFs can significantly clear DPPH in vitro with a dose-depended manner from 0 to $0.15 \mathrm{mg} / \mathrm{mL}$ compatible with the positive drug.

\subsection{Reducing Power Assay}

The reducing power of a compound may serve as a significant indicator of its potential antioxidant activity. The absorbance at $700 \mathrm{~nm}$ was used to demonstrate the reducing power. Increased absorbance of the reaction mixture indicates increased reducing power of the sample. As shown in Figure 2(b), the reducing power of the TFs increased slightly in a dose-depended manner when
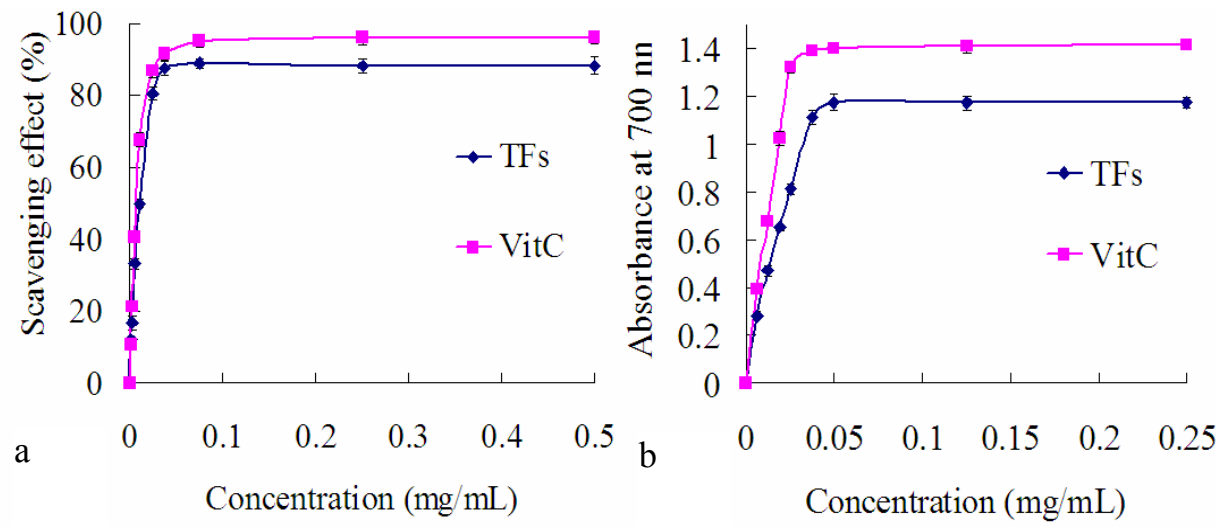

Figure 2. (a) Scavenging effect of the TFs and VitC at different concentrations on DPPH. (b) Reducing power of the TFs and VitC at different concentrations. Data was expressed as means \pm S.D. $(\mathrm{n}=5)$. 
then it reached a plateau. The reducing power of the TFs was 0.812 at $0.025 \mathrm{mg} / \mathrm{mL}$ and 1.17 at $0.125 \mathrm{mg} / \mathrm{mL}$. However, VitC only showed slightly higher activity, with the reducing power of 1.32 at $0.025 \mathrm{mg} / \mathrm{mL}$ and 1.45 at $0.125 \mathrm{mg} / \mathrm{mL}$.

\subsection{Histopathological Assay}

As shown in Figure 3, histopathology of the liver in normal mouse (a) was that the central vein was surrounded by hepatic cord of cells, while the liver of high-fat diet treated mouse (b) showed patches of liver cell necrosis with macrovesicular and microvesicular steatosis, and massive fatty changes. Whereas, the livers of the animals trested by fenofibrate (c) and TFs (E, $50 \mathrm{mg} / \mathrm{kg} /$ day) showed the absence of vesicular steatosis to display good hepatoprotective actions. The low-dose of TFs (d), 25 $\mathrm{mg} / \mathrm{kg} /$ day) treated groups showed less vesicular steatosis.

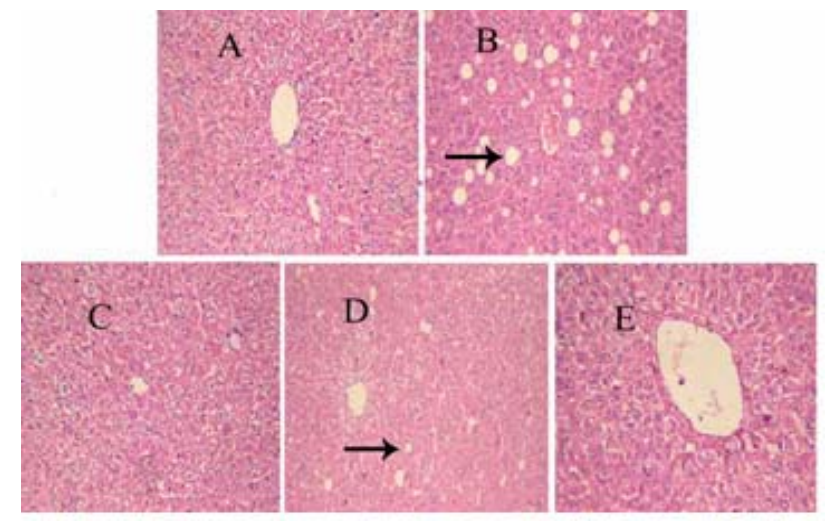

Figure 3. Protective effect of TFs on liver injury induced by high-fat diet in mice. (a) Group I; (b) Group II; (c) Group III; (d) Group IV; (e) Group V. high-fat diet treatment induced vesicular steatosis (black arrows). Hematoxylin and eosin staining; Original magnification, $\times 100$.

Table 1. Effect of TFs on serum lipid profile in experimental animals.

\begin{tabular}{ccccc}
\hline Group & TC $(\mathrm{mg} / \mathrm{dL})$ & TG $(\mathrm{mg} / \mathrm{dL})$ & HDL-C $(\mathrm{mg} / \mathrm{dL})$ & LDL-C $(\mathrm{mg} / \mathrm{dL})$ \\
\hline I & $72.63 \pm 7.22$ & $53.86 \pm 9.19$ & $48.44 \pm 5.49$ & $13.40 \pm 4.27$ \\
II & $143.33 \pm 9.71^{\mathrm{b}}$ & $100.1 \pm 17.3^{\mathrm{b}}$ & $47.03 \pm 4.84$ & $83.55 \pm 14.23^{\mathrm{b}}$ \\
III & $87.12 \pm 7.31^{\mathrm{d}}$ & $64.0 \pm 8.41^{\mathrm{d}}$ & $63.09 \pm 5.49^{\mathrm{c}}$ & $16.18 \pm 7.06^{\mathrm{d}}$ \\
IV & $98.64 \pm 15.74^{\mathrm{c}}$ & $86.18 \pm 4.15^{\mathrm{c}}$ & $64.11 \pm 13.49^{\mathrm{c}}$ & $27.40 \pm 16.53^{\mathrm{d}}$ \\
& & & & \\
V & $78.8 \pm 9.80^{\mathrm{d}}$ & $66.18 \pm 4.75^{\mathrm{d}}$ & $63.84 \pm 13.41^{\mathrm{c}}$ & $18.31 \pm 3.40^{\mathrm{d}}$ \\
\hline
\end{tabular}

Values are given as mean \pm S.D. $(\mathrm{n}=10) .{ }^{\mathrm{a}} p<0.05$ compared with Group I . ${ }^{\mathrm{b}} p<0.01$ compared with Group I. ${ }^{c} p<0.05$ compared with Group II. ${ }^{\mathrm{d}} p<0.01$ compared with Group II.

Table 2. Effect of TFs on serum liver enzymes in experimental animals.

\begin{tabular}{cccc}
\hline Group & ALT (IU/L) & AST (IU/L) & ALP (IU/L) \\
\hline I & $22.70 \pm 3.40$ & $27.70 \pm 5.40$ & $321.92 \pm 130.33$ \\
II & $102.60 \pm 24.50^{\mathrm{b}}$ & $76.50 \pm 17.40^{\mathrm{b}}$ & $495.38 \pm 126.02^{\mathrm{a}}$ \\
III & $45.30 \pm 18.0^{\mathrm{d}}$ & $71.80 \pm 14.86$ & $336.77 \pm 71.15^{\mathrm{c}}$ \\
IV & $91.00 \pm 27.20^{\mathrm{c}}$ & $60.29 \pm 7.90^{\mathrm{c}}$ & $382.28 \pm 83.69^{\mathrm{c}}$ \\
V & $45.8 \pm 2.20^{\mathrm{d}}$ & $54.20 \pm 17.5^{\mathrm{c}}$ & $368.16 \pm 128.08^{\mathrm{c}}$
\end{tabular}

Values are given as mean \pm S.D. $(\mathrm{n}=10) .{ }^{\mathrm{a}} p<0.05$ compared with Group I $.{ }^{\mathrm{b}} p<0.01$ compared with Group I. ${ }^{c} p<0.05$ compared with Group II. ${ }^{\mathrm{d}} p<0.01$ compared with Group II. 


\subsection{Effects of TFs on Serum Lipid Profiles}

As seen in Table 1, the serum levels of TC, TG, and LDL-C were significantly increased in animals fed with high-fat diet compared with those in Group I. The levels of TC, TG and LDL-C of those animals given fenofibrate or TFs were significantly decreased compared with hyperlipemic animals. High-dose of TFs $(50 \mathrm{mg} / \mathrm{kg} /$ day $)$ was able to markedly eliminate TC, TG, and LDL-C by $45.02 \%, 33.86 \%$ and $73.68 \%$, respectively, with no difference compared with the positive group. Although there was no statistically significant difference in the serum HDL-C levels between normal and hyperlipemia mice, an increasing tendency was exhibited in the groups administrated with TFs and fenofibrate.

\subsection{Effect of TFs on Hepatic Enzymes}

The levels of ALT, AST and ALP in serum were performed to evaluate liver function. As can be observed in Table 2, animals fed with high-fat diet exhibited a significantly elevation in serum ALT, AST and ALP compared with normal group, which suggested that the liver was markedly damaged. Compared with hyperlipemic mice, the activities of ALT, AST and ALP were reduced to $55.85 \%, 29.15 \%$ and $25.68 \%$, respectively, of the animals treated by TFs at the dosage of $50 \mathrm{mg} / \mathrm{kg} /$ day, which was no difference compared with fenofibate-treated group. The results implied that the TFs executed a protective effect against liver damage induced by high-fat diet.

\subsection{Effect of TFs on Hepatic Lipid Peroxidation}

Figure 4 showed that the MDA levels of the liver in all experimental groups. The hepatic MDA level of model group was increased significantly compared with normal group $(p<0.01)$. The decline extents of TFs-treated

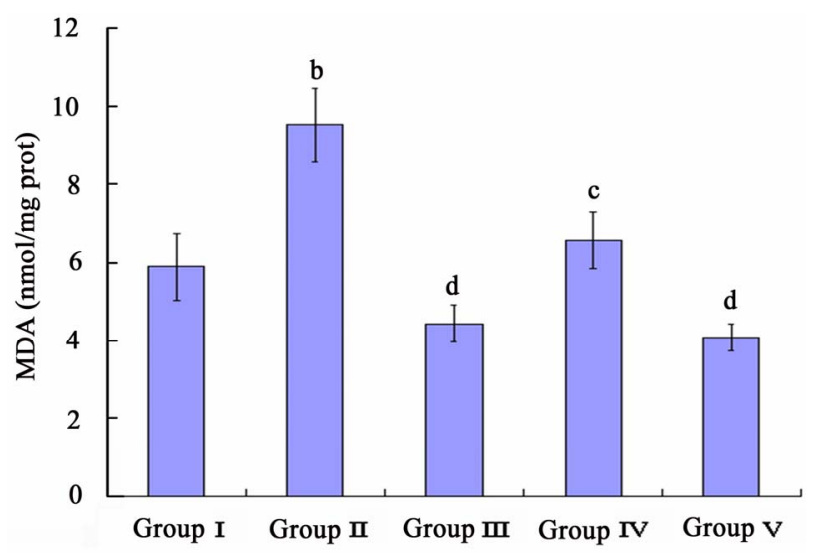

Figure 4. Effect of TFs on hepatic level of MDA in mouse fed with high-fat diet. Values are expressed as mean \pm S.D. $(n=$ 10). ${ }^{\mathrm{b}} p<0.01$ compared with Group I. ${ }^{\mathrm{c}} p<0.05$ compared with Group II. ${ }^{\mathrm{d}} p<0.01$ compared with Group II. groups $(25$ and $50 \mathrm{mg} / \mathrm{kg} /$ day $)$ were $31.09 \%(p<0.05)$ and $53.65 \%(p<0.01)$ compared with hyperlipemic mouse. Meanwhile, there was no difference between fenofibrate- and TFs (50 mg/kg/day)-treated groups.

\subsection{Effect of TFs on Hepatic Antioxidant Enzymes}

The variations of CAT, SOD, GSH and GPX in liver among the experimental groups were shown in Figure 5. Contrasting with normal animals, the levels of CAT, SOD, GSH and GPX of the liver tissues were significantly decreased after feeding high-fat diet for 4 weeks $(p<0.05)$. Fenofibrate and TFs both could amend the antioxidant status in vivo. The improvement capacity of CAT and GPX was TFs $(50 \mathrm{mg} / \mathrm{kg} /$ day $)>$ fenofibate $(50$ $\mathrm{mg} / \mathrm{kg} /$ day $)>$ TFs $(25 \mathrm{mg} / \mathrm{kg} /$ day $)$. Furthermore, the improvement capacities of SOD and GSH were fenofibate $(50 \mathrm{mg} / \mathrm{kg} /$ day $)>$ TFs $(50 \mathrm{mg} / \mathrm{kg} /$ day $)>$ TFs $(25$ $\mathrm{mg} / \mathrm{kg} /$ day).

\section{DISCUSSIONS}

Hyperlipemia is the largest endocrine disease in the world, involving metabolic disorders of carbohydrate, fat and protein. Therefore, it is necessary to search for new drugs that can be used to amendment this metabolic disorder without any side effect. Oxidative stress is currently suggested as a mechanism underlying hyperlipemia, which is one of the major risk factors for coronary artery diseases [21]. In our study, the TFs with high flavonoids showed a good antioxidant activity in vitro, which encouraged us to check the antioxidant activity in vivo in hyperlipemic mice.

MDA, one of the lipid peroxidation products, could make the deomosine between the cellulose molecular relax or inhibit protein synthesis. The hepatic MDA level significantly improved in hyperlipemia animals. In the present experiment, the MDA level was significantly decreased in mouse fed with TFs. These findings are in accordance with those of other investigators [22].

Furthermore, Biological antioxidants are natural compounds which can prevent the un-controlled formation of free radicals and activated oxygen species, or inhibit their reaction with biological structures. These compounds include antioxidative enzymes exist in all oxygen-metabolizing cells, such as superoxide dismutase (SOD), catalase (CAT), glutathione peroxidase (GPX) and non-enzymatic antioxidants, such as glutathione (GSH), vitamin C and vitamin $\mathrm{E}$ [23]. The main role of the CAT, mainly existing in some cells, is to catalytic the decomposition of hydrogen peroxide [24]. It could prevent hydrogen peroxide to form hydroxyl radical, which is the most harmful radical in vivo [25]. SOD mutates the superoxide radicals to form molecular oxygen and $\mathrm{H}_{2} \mathrm{O}_{2}$. GPX, one of the most important hepatic detoxification 

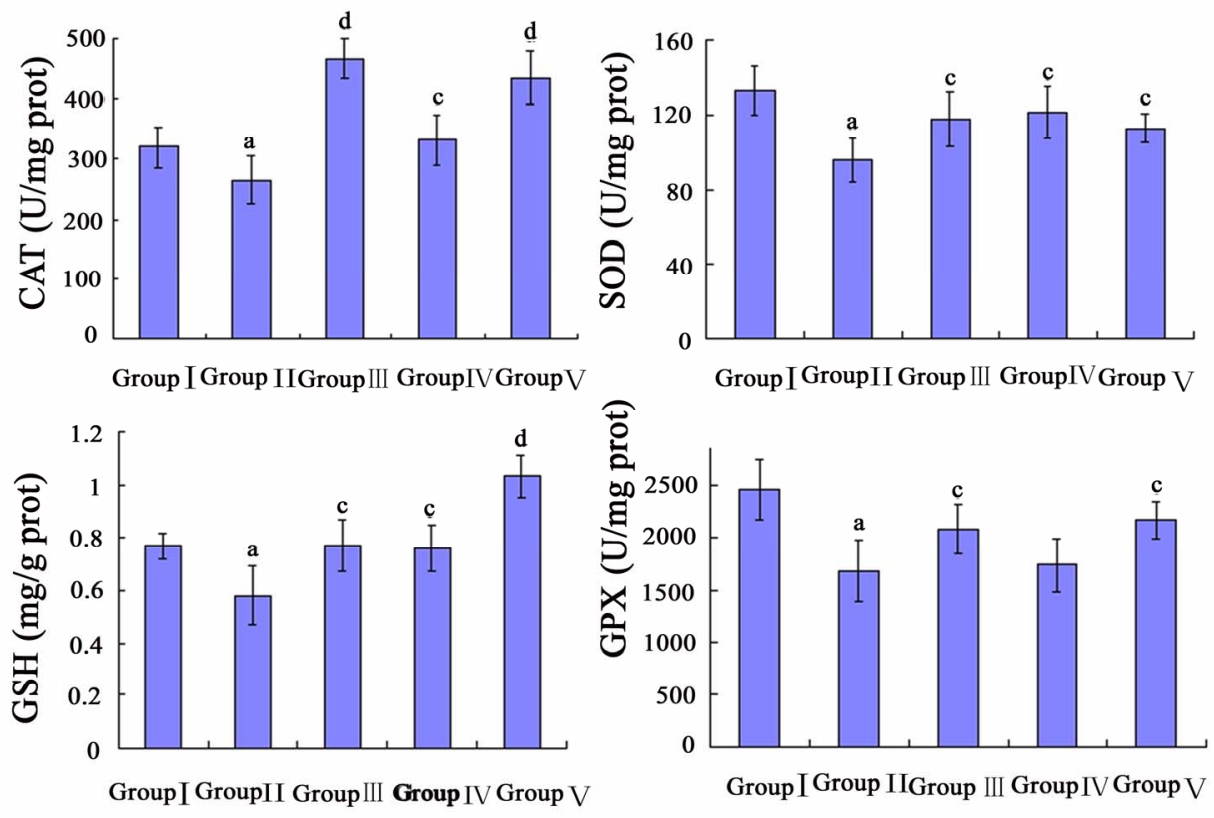

Figure 5. Effect of TFs on hepatic levels of CAT, SOD, GSH and GPX in mouse fed with high-fat diet. Values are expressed as mean \pm S.D. $(\mathrm{n}=10) .{ }^{\mathrm{a}} p<0.05$ compared with Group I $.{ }^{\mathrm{b}} p<0.01$ compared with Group I. ${ }^{\mathrm{c}} p<0.05$ compared with Group II. ${ }^{\mathrm{d}} p<0.01$ compared with Group II.

elements [26], almost presents in the whole biological tissues, especially the liver and RBC. It can scavenge lipid peroxidation induced by hydroxyl radical, postpone the aging of cells and clear the nucleic acid peroxide. Moreover, GPX could remove hydrogen peroxide generating in the tissues. Meanwhile, GSH is the most important biomolecule against chemically induced toxicity and can participate in the elimination of reactive intermediates by reducing hydroperoxides in the presence of GPX $[27,28]$. GSH also functions as a free radical scavenger and in the repair of free radical-induced biological damages [28]. The decrease in the GSH level represents increased utilization due to oxidative stress [29].

The efficiency of this defense system is apparently weakened in hyperlipemia condition resulting in ineffective scavenging of free radicals and lipid peroxidantion products, which can improve the levels of tissue lipid profiles in vivo. The inhibition of CAT, SOD and GPX activities are found to be involved in many degenerative diseases. Likewise, in our study, high-fat diet caused a markedly decrease in SOD, GPX, CAT activities and nonenzymatic antioxidant (GSH) levels in test organs in mice. These adaptive decreases in antioxidant enzyme activities protect the organs against lipid peroxidation mouse [30]. TFs significantly increased hepatic CAT, SOD, GSH and GPX levels. Our data also showed that TFs significantly reduced the hepatic MDA concentration in a dose-dependent manner. These results suggested that, the antioxidative effect of TFs might reduce oxidative stress, resulting in a lower lipid peroxidation in liver. These results indicated that the antioxidative effect of TFs in the liver may only be observed in the presence of severe oxidative stress, suggesting that TFs may act as a chemopreventive agent with inhibition activity on oxidative-induced cell damage.

Elevated blood triglyceride and cholesterol, especially low-density-lipoprotein cholesterol (LDL-C), is a major risk factor in development of cardiovascular disease [31]. It is well-known that cholesterol is a fatty substance which is important to the membrane of cells in the animal body. TC is a measure of the total amount of all cholesterol in blood at a given time and is the sum of HDL-C, LDL-C. TG composed of three fatty acids and glycerol. Liking cholesterol, they circulate in blood, but are stored in body fat and used when body needs extra energy. HDL-C removes excess cholesterol from arteries and moves it to liver for further processing or to be eliminated from the body. The higher serum HDL-C is the better. Therefore, the HDL-C is called "good" cholesterol. It also plays a key role in the protection against oxidative damage of membrane. LDL-C contributes to buildup of fat deposits in the arteries (atherosclerosis), which can cause decreased blood flow and head attack. So it is always called "bad" cholesterol, and a less levels are desirable. The value of AI indicates the deposition of foam cells or plaque or fatty infiltration or lipids in heart, coronaries, aorta, liver and kidney. In this study, TC, TG and LDL-C levels significantly increased in the hyperlipemic animals fed a high-fat diet for 4 weeks, but with no significantly decreased in HDL-C level (Table 1). All these results showed that the mouse hyperlipemic model was established successfully by feeding a high-fat 
diet for 4 weeks. After the experiment, the animals administration of fenofibrate and TFs were all exhibited a decrease tendency $(p<0.05)$. As to the data of HDL-C in the experiment, there was also a remarkable increase of the serum HDL-C levels in the mouse fed a high-fat diet for 4 weeks. The increase of 'good' cholesterol does not mean high-fat diet is favorable to serum lipid profile levels, because the increase of 'good' probably does not surpass that of 'bad', which has negative effects on lipid-lowering. In summary, the administration of TFs could decrease the serum levels of TC, TG and LDL-C.

The liver is capable of removing cholesterol from the blood circulation as well as manufacturing cholesterol and secreting cholesterol into the blood circulation and liver damages are generally induced in the condition of hyperlipemia as dramatic increase of serum ALT and AST levels. Furthermore, ALP, one of hepatic enzymes, also reflects the damage induced by high-fat diet. In our test, the damage to liver induced by high-fat diet was also investigated, which included hepatic enzymes and histopathological examination. These results indicated that TFs was characterized by an ameliorating effect on fatty liver.

\section{CONCLUSIONS}

The present study demonstrated TFs from $R$. laevigata Michx has favorable potency to develop a hypolipidemic and hepatoprotective activities, of which the levels may be mediated, in part, by enhancing the system of antioxidant defense. It can be used as a potential medicine for cardiovascular diseases. The toxicity, clinical application, and chemical constituents of TFs are not clear and need further investigation.

\section{ACKONWLEDGEMENT}

This research was supported by funds of Liaoning BaiQianWan Talents Program, Liaoning, China.

\section{REFERENCES}

[1] Frishman, W.H. (1998) Biologic Markers as Predictors of Cardiovascular Disease. The American Journal of Medicine, 104(6A), 18S-27S.

[2] Williams, G. and Pickup, J. Ed., (1991) New drugs in the management of diabetes mellitus. Textbook of Diabetes II. Blackwell, Oxford, 977-993.

[3] Kameswara Rao, B., Kesavulu, M.M., Giri, R. and Apparao, Ch. (1999) Antidiabetic and hypolipidemic effects of Momordica cymbalaria Hook. Fruit powder in alloxan diabetic rats. Journal of Ethnopharmacology, 67(1), 103-109.

[4] Sharma, B., Balomajumder, C. and Roy, P. (2008) Hypoglycemic and hypolipidemic effects of flavonoid rich ex- tract from Eugenia jambolana seeds on streptozotocin induced diabetic rats. Food Chemisty Toxicology, 46(7), 2376-2383.

[5] Dini, I., Tenore, G.C. and Dini, A. (2009) Saponins in Ipomoea batatas tubers: Isolation, characterization, quantification and antioxidant properties. Food Chemistry, 113(2), 411-419.

[6] Ma, M., Liu, G.H., Yu, Z.H., Chen, G. and Zhang, X. (2009) Effect of the Lycium barbarum polysaccharides administration on blood lipid metabolism and oxidative stress of mice fed high-fat diet in vivo. Food Chemistry, 113(4), 872-877.

[7] Wang, J.Q., Li, J., Zou, Y.H., Cheng, W.M., Lu, C., Zhang, L., Ge, J.F., Huang, C., Jin, Y., Lv, X.W., Hu, C.M. and Liu, L.P. (2009) Preventive effects of total flavonoids of Litsea coreana leve on hepatic steatosis in rats fed with high fat diet. Journal of Ethnopharmacology, 121(1), 54-60.

[8] Zhang, T.Y., Nie, L.W., Wu, B.J., Yang, Y., Zhao, S.S., and Jin, T. (2004) Hypolipedemic activity of the polysaccharose from Rosa laevigata Michx fruit. Chinese Journal of Public Health, 20(7), 829-830.

[9] Welton, A.F., Hurley, J. and Will, P. (1988) Flavonoids and arachidonic acid metabolism. Progress in Clinical and Biological Research, 280, 301-312.

[10] Sharma, B., Balomajumder, C. and Roy, P. (2008) Hypoglycemic and hypolipidemic effects of flavonoid rich extract from Eugenia jambolana seeds on streptozotocin induced diabetic rats. Food Chemistry and Toxicology, 46(7), 2376-2383.

[11] Diouf, P.N., Stevanovic, T. and Cloutier, A. (2009) Study on chemical composition, antioxidant and anti-inflammatory activities of hot water extract from Picea mariana bark and its proanthocyanidin-rich fractions. Food Chemistry, 113(4), 897-902.

[12] Xu, S.Y., Bian, R.L. and Chen, X. Ed., (2002) Experimental methodology of pharmacology. People' Medical Publishment House, Beijing, 1202.

[13] China Pharmacopoeia Committee. (2005) Pharmacopoeia of the People's Republic of China, the first division of 2005 edition Ed., 291-292, China Chemical Industry Press. Beijing.

[14] Hsu, B., Coupar, I.M., and Ng, K. (2006) Antioxidant activity of hot water extract from the fruit of the Doum palm, Hyphaene thebaica. Food Chemistry, 98(2), 317-328.

[15] Tsai, S.Y., Huang, S.J. and Mau, J.L. (2006) Antioxidant properties of hot water extracts from Agrocybe cylindracea. Food Chemistry, 98(4), 670-677.

[16] Lowry, O.H., Rosebrough, N.J. and Far, A.L. (1951) Protein measurement with the Folin phenol reagent. Journal of Biological Chemistry, 193(1), 265-275.

[17] Friedwald, W.T., Levy, R.J. and Fredricken, D.S. (1972) Estimation of HDL-C in the plasma without the use of preparative ultracentrifuge. Clinical Chemistry, 18, 449.

[18] Fu, B., Liu, J., Li, H., Li, L., Lee, F.S.C. and Wang, X. (2005) The application of macroporous resins in the separation of licorice flavonoids and glycyrrhizic acid. Journal of Chromatography A, 1089(1-2), 18-24.

[19] Peng, J.Y, Yang, G.J., Fan, G.R. and Wu, Y.T. (2005) Preparative isolation and separation of a novel and two 
known flavonoids from Patrinia villosa Juss by highspeed counter-current chromatography. Journal of Chromatography A, 1092(2), 235-240.

[20] Soares, J.R., Dins, T.C, Cunha, A.P. and Ameida, L.M. (1997) Antioxidant activity of some extracts of Thymus zygis. Free Radical Research, 26, 469-478.

[21] Fruchart, J.C. and Duriez, P. (1998) High-density lipoproteins and coronary heart disease future prospects in gene therapy. Biochimie, 80(2), 167-172.

[22] Mahfouz, M.M. and Kummerow, F.A. (2000) Cholesterol-rich diets have different effect on lipid peroxidation, cholesterol oxides, and antioxidant enzymes in rats and rabbits. The Journal of Nutritional Biochemistry, 11(5), 293-302.

[23] Fridovich, I. (1999) Fundamental aspects of reactive oxygen species, or what's the matter with oxygen? The New York Academy of Sciences, 893(1), 13-18.

[24] Yao, D.C., Shi, W.B., Gou, Y.L., Zhou, X.R., Tak, Y.A. and Zhou, Y.K. (2005) Fatty acidmediated intracellular iron translocation: A synergistic mechanism of oxidative injury. Free Radical Biology and Medicine, 39, 1385-1398.

[25] Chance, B., Sies, H. and Boveris, A. (1979) Hydroperoxide metabolism in mammalian organs. Physiological Reviews, 59(3), 527-605.

[26] Shaw, S., Rubin, K. and Lieber, C.S. (1986) Depressed hepatic glutathione and increased diene conjugates in alcoholic liver disease: Evidence of lipid peroxidation. $\mathrm{Di}$ gestive Diseases and Sciences, 28, 585-589.

[27] Nicotera, P. and Orrenius, S. (1986) Role of thiols in protection against biological reactive intermediates. Advances in Experimental Medicine and Biology, 197, 41-51.

[28] Lin, T. and Yang, M.S. (2007) Benzo [a] pyrene-induced elevation of GSH level protects against oxidative stress and enhances xenobiotic detoxification in human HepG2 cells. Toxicology, 235(1-2), 1-10.

[29] Anuradha, C.V. and Selvam, R. (1993) Effect of oral methionine on tissue lipid peroxidation and antioxidants in alloxan-induced diabetic rats. The Journal of Nutritional Biochemistry, 4(4), 212-217.

[30] Padmavathi, R., Senthilnathan, P., Chodon, D. and Sakthisekaran, D. (2006) Therapeutic effect of paclitaxel and propolis on lipid peroxidation and antioxidant system in 7, 12 dimethyl benz (a) anthracene-induced breast cancer in female Sprague Dawley rats. Life Science, 78(24), 2820-2825.

[31] Anderson, J.W. and Tietyen-Clark, J. (1986) Dietary fiber: Hyperlipidemia, hypertension, and coronary heart disease. The American Journal of Gastroenterology, 81(10), 907-919. 